\title{
Observational Study of Buprenorphine Treatment of Opioid-Dependent Pregnant Women in a Family Medicine Residency: Reports on Maternal and Infant Outcomes
}

\author{
Alane O'Connor, DNP, William Alto, MD, MPH, Katje Musgrave, DO, \\ Daniel Gibbons, DO, Lalaine Llanto, MD, Selma Holden, MD, and \\ Jonathan Karnes, MD
}

Purpose: Within a family medicine residency, an outpatient buprenorphine treatment program was provided for pregnant women who were dependent on opioids. The purpose of this study was to investigate the outcomes of infants who were exposed to a range of doses of buprenorphine in utero and to determine how closely observed maternal/fetal outcomes (eg, method of delivery and infants requiring treatment for neonatal abstinence syndrome [NAS]) match those previously reported in the literature.

Methods: This study consists of a retrospective case series of 23 infants born to 22 pregnant women who were dependent on opioids and who were treated with buprenorphine during a 31-month period.

Results: Thirty-five percent of infants $(8$ of 23$)$ required treatment for NAS. There was no significant relationship between maternal dose of buprenorphine and birth weight, NAS severity (ie, peak NAS score), or time to first peak NAS score. Infants born to tobacco users had a significantly lower birth weight $(P=.0136)$ than infants born to nonusers. Seventy percent of infants $(16$ of 23$)$ were breastfeeding at the time of discharge from the hospital.

Conclusions: The observations made in this case series are consistent with data previously reported in the literature. Infants in this study were more likely to be breastfed than those previously described by others, but further analysis is necessary to determine whether this finding is significant. ( $\mathrm{J}$ Am Board Fam Med 2011;24:194-201.)

Keywords: Observational Study, Opioids, Prenatal Care, Buprenorphine, Neonatal Abstinence Syndrome

Estimates of illicit substance use during pregnancy are highly variable and range from $0.4 \%$ to $27 \%$ among all pregnant women in the United States. ${ }^{1}$ Opioid abuse during pregnancy continues to present a major public health concern. ${ }^{2}$ Early identification and treatment of opioid-dependent pregnant women is essential because it reduces potential complications from recurrent intoxication and

This article was externally peer reviewed.

Submitted 5 July 2010; revised 30 November 2010; accepted 7 December 2010.

From the Maine Dartmouth Family Medicine Residency, Augusta.

Funding: none.

Conflict of interest: none declared.

Corresponding author: Alane O'Connor, DNP, Maine Dartmouth Family Medicine Residency, 15 East Chestnut Street, Augusta, ME 04330 (E-mail: aoconnor@mainegeneral.org). withdrawal from opioids for both the fetus and the mother. It also helps to stabilize the woman's social situation, reduces the risk of maternal overdose of opioids, and reduces the risk of communicable blood-borne diseases associated with intravenous drug use. The use of prescribed buprenorphine at regular intervals reduces the variability of the maternal serum opioid levels that accompany recurrent use of short-acting opioids, thereby reducing intrauterine fetal withdrawal. ${ }^{3}$ The maternal-fetal effects of opioid dependence during pregnancy range from no anomalies or adverse outcomes to intrauterine growth restriction, placental insufficiency, preeclampsia, preterm rupture of membranes, premature delivery, postpartum hemorrhage, and perinatal mortality. ${ }^{1,4}$ Fetal withdrawal from opioids in nonhuman subjects is associated 
with increased morbidity and mortality, likely because of reduced intrauterine and placental blood flow and diminished availability of oxygen to the fetus. $^{5}$

Methadone is a highly efficacious, evidencebased treatment and continues to be the standard of care for opioid-dependent pregnant women. ${ }^{6}$ However, the Center for Substance Abuse Treatment recommends the use of buprenorphine when the benefits outweigh the risks, when access to a methadone clinic is not available, or when the patient refuses methadone treatment. ${ }^{7}$ Buprenorphine monotherapy should be used during pregnancy because the effects of fetal exposure to sublingual naloxone used in the buprenorphinenaloxone combination tablets have not been studied. ${ }^{7}$ Monotherapy also is preferred because exposure to naloxone in utero may produce hormonal changes in the fetus, and the intravenous misuse of the combination buprenorphine-naloxone tablets may produce acute withdrawal. ${ }^{8}$ However, it is important to note that a greater risk of diversion exists for those taking buprenorphine monotherapy, so these patients should be monitored frequently.

The use of buprenorphine rather than methadone during pregnancy offers many benefits. Buprenorphine may be more convenient for patients because it can be dispensed in the primary care setting rather than requiring daily visits to a specialized clinic, thereby potentially reducing the stigma associated with treatment and the frequency of social contacts with other opioid-dependent persons. When compared with infants born to mothers maintained on methadone, buprenorphine-exposed neonates required one-third as much medication to treat neonatal abstinence syndrome (NAS) and remained in the hospital for a significantly shorter time (1.3-day difference). ${ }^{9}$ The severity and course of NAS was significantly longer ( $>1$ week) and more severe (mean Finnegan score of 17.8 for infants who were exposed to methadone compared with 9.2 for buprenorphine) for infants whose mothers were maintained on methadone during pregnancy when compared with infants whose mothers were maintained on buprenorphine. ${ }^{10}$ Compared with methadone, treatment with buprenorphine seems to attenuate both the severity and duration of NAS, but more rigorous, casecontrolled studies are needed to confirm this finding. ${ }^{11}$

\section{Methods}

This case series was derived from a maternal-infant opioid treatment program integrated into a family medicine residency serving a predominantly rural and underserved population. The program included an intensive, holistic treatment plan that provided prenatal and obstetric care, addiction treatment and therapy, care for the mother and child during and after delivery, and social services support. Two faculty and 6 resident physicians provided continuity care for these families and were supported by a psychologist and social worker. This study examines the maternal/fetal outcomes (eg, method of delivery, infants requiring treatment for NAS) of opioid-dependent pregnant women compared with previously reported data. The study also compares the outcomes of infants who were exposed to a range of doses of buprenorphine in utero with those reported in the literature.

All pregnant women in this case series had a Diagnostic and Statistical Manual of Mental Disorders IV, Text Revision, diagnosis of current opioid dependence and requested maintenance therapy with buprenorphine. Women were informed that methadone continues to be the standard of care in the treatment of opioid-dependent pregnant women. Urine toxicology screens and opioid confirmation tests were done during intake for all women and at each clinic visit for medical or obstetric care or group counseling. Additional confirmation tests were performed if other illicit drugs were detected on the toxicology screen and the women denied having used the illicit substance.

Consistent with hospital policy, infants born to opioid-dependent women were observed for NAS in the hospital setting for at least 5 days after birth. Among other symptoms, NAS can include respiratory distress, sneezing, yawning, mottling, feeding problems, tremor, high-pitched cry, and increased muscle tone. ${ }^{12}$ NAS usually begins within 72 hours of birth and can last for several weeks. ${ }^{12}$ The Finnegan Scoring System, a quantitative tool that includes 31 of the most commonly observed NAS symptoms, was used to measure the degree to which an infant was experiencing symptoms associated with opioid withdrawal. ${ }^{13}$ A degree of severity was assigned to each score. Pharmacologic intervention was indicated when 3 or more consecutive NAS scores were $\geq 8$, when the average of 3 consecutive scores was $\geq 8$, when 2 or more consecutive NAS 
scores were $\geq 12$, or when the average of 2 or more consecutive NAS scores was $\geq 12$. Infants who required treatment for NAS were given phenobarbital as a first-line medication.

To identify studies for comparison, a Medline search of original research conducted on humans and reported in English from 1980 to 2010 was performed using the keywords "pregnancy," "buprenorphine," and "NAS." Descriptive statistics including mean, range, and SD were used to describe the maternal and infant populations. Given the small sample sizes, inferential statistics were used to a limited extent. Regression modeling, simple correlations, and independent sample $t$ tests were completed using STATA software version 10 (StataCorp, LP, College Station, TX). Results were considered to be statistically significant when $P \leq$ .05 . The institutional review board of Maine General Medical Center approved this study.

\section{Results}

The office and hospital charts of 27 pregnancies among opioid-dependent women who were treated with buprenorphine from August 2007 until February 2010 were reviewed. Two women who were initially treated with buprenorphine but had a spontaneous or therapeutic abortion before 12 weeks were excluded from the study. Two women who initiated treatment with buprenorphine but ultimately switched to methadone before delivery were also excluded; after transferring to methadone, these women chose to have their obstetric care provided elsewhere, so their outcomes were not available for comparison. None of the women in this series experienced an unplanned pregnancy loss after 12 weeks.

Twenty-three pregnancies among opioid-dependent pregnant women who were treated with buprenorphine met the inclusion criteria of this case series (22 women and 23 infants [one woman had 2 separate pregnancies during the 31-month period]). Three of these women were prescribed buprenorphine/naloxone before pregnancy, whereas the remainder initiated treatment during the course of their pregnancy. Of the women who initiated treatment during pregnancy, all but 3 had their dosage titrated by clinicians in this family medicine residency. Dosage was titrated to control both maternal withdrawal symptoms and cravings for other opioids. Two women transferred care to this prac- tice from drug rehabilitation facilities, and one was prescribed buprenorphine by a clinician outside of this program until she was 35 weeks pregnant. With the exception of one patient who initiated both buprenorphine treatment and obstetric care at 20 weeks, the remaining women were being treated with buprenorphine by 13 weeks' gestation.

The mean gravida and parity of the women in this case series was 2.7 (range, 1-7) and 1.2 (range, $0-3)$, respectively. Their mean age at delivery was 25.8 years (range, 20.7-33.8 years; SD, 3.62 years). During 3 of the 23 pregnancies (13\%) the women married, and during 18 of the 23 pregnancies (78\%) the women were living with the father of this infant. With the exception of 2 patients, all the women had Medicaid as their primary health insurance. All but 5 of the women (78\%) had concomitant psychological diagnoses, including anxiety, depression, attention deficit hyperactivity disorder, bipolar disorder, and posttraumatic stress disorder. None tested positive for HIV.

Characteristics of the mothers and infants are presented in Table 1 . The mean daily buprenorphine dose of the women in this series during their first trimester was $7.9 \mathrm{mg}$ (range, 2-16 mg). During the second trimester, the mean daily dose increased to $11.1 \mathrm{mg}$ (range, $3-16 \mathrm{mg}$ ). At delivery the mean dose was $13.3 \mathrm{mg}$ (range, 3-24 mg). During 16 of the 23 pregnancies $(70 \%)$ the women required dose increases. Among all women, the mean dose increase over the course of pregnancy was $5.9 \mathrm{mg}$.

During 15 of the 23 deliveries (65\%), the women required epidural anesthesia. Seven of the 23 (30\%) delivered by cesarean section whereas the remainder delivered vaginally (one required instrumented delivery). Three of the cesarean sections were needed because of a failure to progress, 2 were repeat cesareans, one was needed because of cephalopelvic disproportion, and one was elective because of maternal concern about the transmission of the hepatitis $\mathrm{C}$ virus. There were no instances of fetal distress. Average APGAR (appearance, pulse, grimace, activity, respiration) scores were 8.3 (range, 7-9) and 9.0 (range, 8-9) at 1 and 5 minutes after birth, respectively. Twenty of 23 infants (87\%) were delivered at term; the remaining mothers delivered at 36 weeks, $363 / 7$ weeks, and 36 6/7 weeks). The average birth weight was $3148 \mathrm{~g}$ (range, 2320$4295 \mathrm{~g}$; SD, $471 \mathrm{~g})$.

Eight of the 23 infants (35\%) required treatment for NAS. The mean time to first peak NAS score 
Table 1. Characteristics of Maternal and Infant Populations

\begin{tabular}{|c|c|c|c|c|c|c|c|}
\hline & $\begin{array}{c}\text { Birth } \\
\text { Weight (g) }\end{array}$ & $\begin{array}{l}\text { Maternal Daily } \\
\text { Dose of } \\
\text { Buprenorphine at } \\
\text { Delivery (mg) }\end{array}$ & $\begin{array}{l}\text { NAS Severity } \\
\text { (Peak NAS } \\
\text { Score) }\end{array}$ & $\begin{array}{c}\text { Required } \\
\text { Treatment for } \\
\text { NAS }\end{array}$ & $\begin{array}{l}\text { Days in } \\
\text { Hospital }\end{array}$ & $\begin{array}{l}\text { Time to First } \\
\text { Peak NAS } \\
\text { Score (hr) }\end{array}$ & $\begin{array}{c}\text { Tobacco Use } \\
\text { during } \\
\text { Pregnancy }\end{array}$ \\
\hline & 2525 & 16 & 10 & No & 8 & 103.5 & Yes \\
\hline & 2772 & 10 & 5 & No & 7 & 65 & Yes \\
\hline & 3745 & 24 & 5 & No & 6 & 16 & No \\
\hline & 3110 & 16 & 10 & No & 7 & 105 & Yes \\
\hline & 2929 & 12 & 13 & No & 6 & 104 & Yes \\
\hline & 3487 & 4 & 8 & No & 6 & 94.3 & Yes \\
\hline & 3025 & 12 & 14 & Yes & 9 & 143.3 & Yes \\
\hline & 3515 & 6 & 11 & No & 10 & 41 & Yes \\
\hline & 3170 & 16 & 12 & Yes & * & 38 & No \\
\hline & 3855 & 24 & 8 & No & 7 & 1 & Yes \\
\hline & 3115 & 16 & 8 & No & 6 & 88.3 & Yes \\
\hline & 3025 & 12 & 9 & No & 6 & 11.3 & Yes \\
\hline & 3530 & 16 & 14 & Yes & 13 & 36 & Yes \\
\hline & 3080 & 8 & 4 & No & 6 & 52 & Yes \\
\hline & 2320 & 16 & 11 & Yes & 7 & 71 & Yes \\
\hline & 3455 & 12 & 16 & Yes & * & 39 & Yes \\
\hline & 2885 & 16 & 19 & Yes & 11 & 114 & Yes \\
\hline & 2778 & 6 & 18 & Yes & 16 & 38 & Yes \\
\hline & 2870 & 16 & 11 & No & 6 & 69.3 & Yes \\
\hline & 3415 & 12 & 9 & No & 6 & 86 & No \\
\hline & 4295 & 16 & 10 & No & 6 & 29.3 & No \\
\hline & 2400 & 16 & 6 & No & 5 & 58 & Yes \\
\hline & 3095 & 3 & 9 & Yes & 7 & 120 & Yes \\
\hline Mean (SD) & $3148(471.0)$ & $13.3(5.4)$ & $10.4(3.9)$ & & $7.7(2.7)$ & $66.2(38.4)$ & \\
\hline
\end{tabular}

*Infants were transferred to another hospital; these infants are not included in mean number (SD) of days in hospital.

NAS, neonatal abstinence syndrome.

for infants who required treatment was 74.9 hours (SD, 44.4 hours) compared with 61.6 hours (SD, 35.6 hours) for the 15 infants who did not require treatment. There was no significant relationship between time to first peak NAS score and NAS severity (ie, peak NAS score), as illustrated in Figure 1. For all infants the mean time to first peak NAS score was 66.2 hours (SD, 38.4 hours).

There was no statistically significant relationship between the maternal dose of buprenorphine and the severity of NAS. There was no significant difference in the mean maternal buprenorphine dose among infants who required treatment for NAS $(12.1 \mathrm{mg})$ and the mean maternal dose of infants who did not require treatment for NAS $(13.9 \mathrm{mg})$. There was no significant relationship between the maternal dose of buprenorphine and either birth weight or time to first peak NAS score, and there was no significant difference in NAS severity of infants born to mothers who were taking buprenor- phine before pregnancy (mean peak NAS score, 10.3) and infants born to mothers who initiated treatment during the course of their pregnancy (mean peak NAS score, 10.5).

There was no significant relationship between daily tobacco consumption and either NAS severity or time to first peak NAS score. Although the mean NAS severity score increased as daily tobacco consumption increased (9.0 for nonsmokers, 9.3 for women smoking 4 to 5 cigarettes daily, and 10.8 for women smoking $\geq 10$ cigarettes daily), the relationship was not statistically significant. Infants born to tobacco users had a significantly lower birth weight $(P=.0136)$ than infants born to nonusers.

Seventy percent of infants (16 of 23) were breastfeeding at discharge from the hospital and 50\% (11 of 22) were still breastfeeding at the 6- to 8 -week follow-up visit (data for one infant was not available).

Meconium drug screens (performed by the Mayo Clinic) were completed on 20 of the 23 
Figure 1. Relationship between NAS severity and time to peak NAS score.

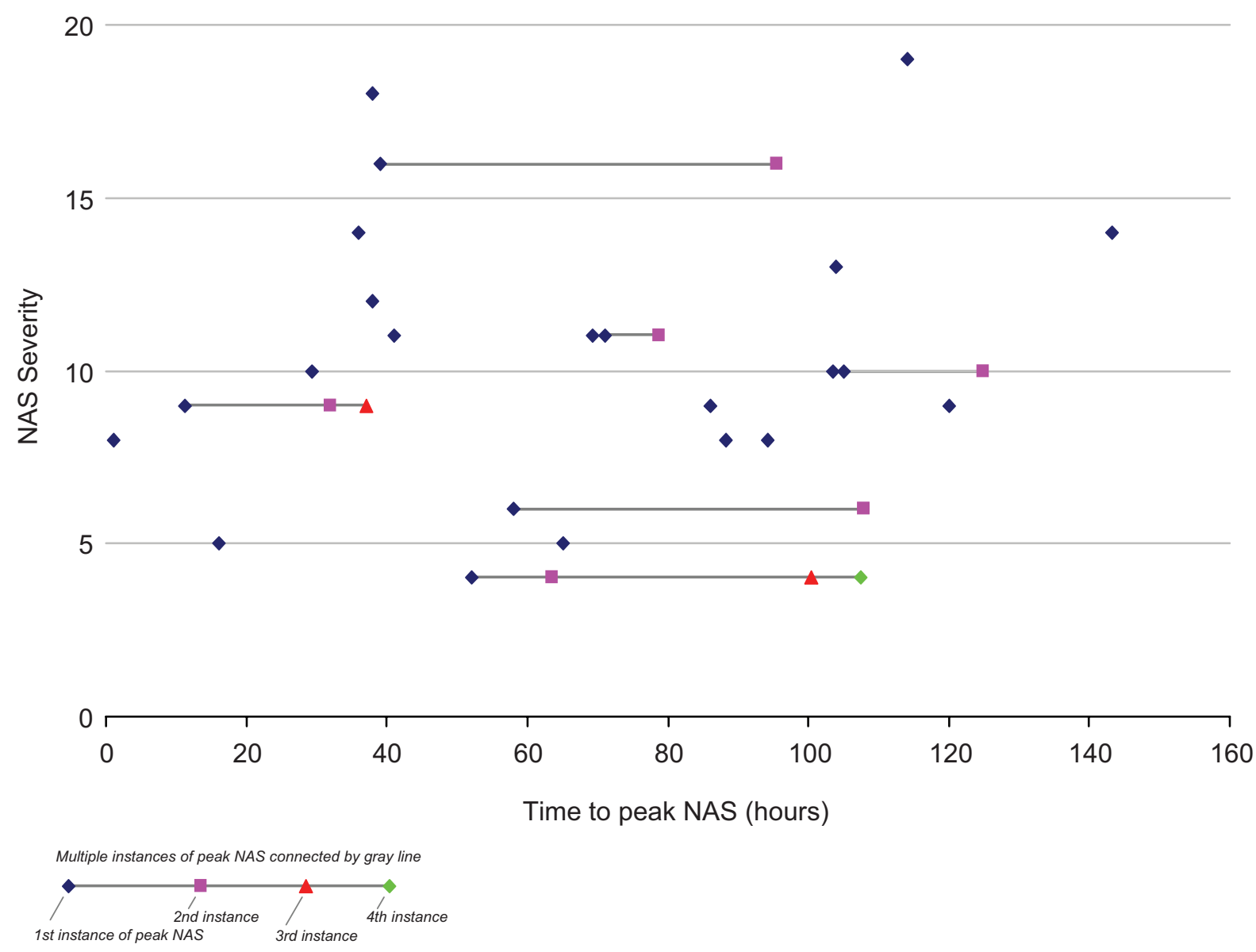

infants, of which only 5 were positive ( 4 for marijuana and one for hydrocodone). During 9 of the 23 pregnancies $(39 \%)$ the women tested positive for illicit opioids on urine testing. The women continued to smoke tobacco during $83 \%$ of pregnancies (19 of 23) and continued to smoke marijuana during $35 \%$ of pregnancies ( 8 of 23 ). Only one woman admitted to using alcohol during pregnancy. Nonprescribed stimulants, including amphetamines and/or cocaine, were used during 2 of the 23 pregnancies $(9 \%)$, whereas one woman was taking prescribed amphetamines during her first trimester until it was determined that she was pregnant. Nonprescribed benzodiazepines were used during 5 of the 23 pregnancies (22\%). Three mothers took prescribed antidepressant medications during their pregnancies $(13 \%)$ and one woman was taking a prescribed antipsychotic medication.

All of the infants born to the pregnant women in this series were discharged with their mothers after observation and treatment for NAS as indicated.
Only one woman was lost to follow-up after delivery.

\section{Discussion}

Relatively few studies of women who were treated with buprenorphine during pregnancy and their infants are available for comparison, and many of the existing studies have smaller sample sizes than this series (eg, 9, 10,13, 15, 31, 67, and 158 opioiddependent pregnant women and/or their infants were maintained on buprenorphine). ${ }^{3,9,14-18}$

Because of the physiologic changes or psychological stressors of pregnancy, many women required buprenorphine dose increases to control cravings for other opioids during the course of their pregnancy. Other authors found a 3.3-mg (range, $0-6 \mathrm{mg}$ ) average increase in maternal dose of buprenorphine over the course of a pregnancy and a slight increase $(0.5 \mathrm{mg})$ in the mean dose of buprenorphine taken during the last trimester of 
pregnancy. ${ }^{9,14}$ Sixteen women in this case series (70\%) required buprenorphine dose increases during the course of their pregnancy. The mean dose increase during pregnancy was $5.9 \mathrm{mg}$, and the mean dose increase during the third trimester was $2.5 \mathrm{mg}$.

Caring for opioid-dependent pregnant women during labor can be challenging because buprenorphine's high affinity for the $\mu$ receptors may block the action of many commonly used analgesic medications. The doses of buprenorphine that are prescribed to treat opioid dependence are inadequate for the treatment of labor pain. Epidural anesthesia can be used effectively, but commonly used antagonist medications such as nalbuphine or butorphanol should be avoided because they can precipitate acute withdrawal in the buprenorphine-dependent patient. ${ }^{8}$ A small study of 8 women maintained on buprenorphine during pregnancy suggests that opioid analgesics also can be used to treat postpartum pain, but that treatment must be individualized. ${ }^{19}$ The authors concluded that "there is considerable individual variability in response to these medications... [and] buprenorphine patients do respond to additional opioid medications given for pain control." ${ }^{19}$ Sixty-five percent of the women in this case series required epidural anesthesia, which is similar to previously reported data $(60 \%) .{ }^{18}$

Thirty-five percent of deliveries (8 of 23) required either instrumented delivery or Cesarean section, which is higher than previously reported $(19.5 \%) .{ }^{18}$ Thirteen percent (3 of 23) of infants in this case series were delivered before 37 weeks, which is in the range of previously reported data (4.5\%, 10\%, 25\%). ${ }^{14,17,18}$ The mean birth weight of infants in this series was $3148 \mathrm{~g}$, which is in the range of previously reported data $(2796,2843$, $3000,3049$, and $3180 \mathrm{~g}){ }^{3,15-18}$ Similarly, the mean gestational age of infants was 39.3 weeks compared with 38.4 weeks, 38.8 weeks, and 39.6 weeks, which have been reported previously. ${ }^{3,16,18}$ The mean time to first peak NAS score for all infants in this case series was 66.2 hours, which is similar to existing data. ${ }^{14,18}$

Thirty-five percent of infants ( 8 of 23 ) required treatment for NAS, which is within the range of previous studies $(14.9 \%, 25.8 \%, 52 \%, 57 \%$, $62.5 \%$, and $76.9 \%) .{ }^{14-18,20}$ The mean peak NAS score for infants in this series was 10.4 (SD, 3.9; range, 4-19), which is also similar to previously reported data (mean score of 11.0; SD, 3.6; range, $5-16) .{ }^{16}$ There was no difference between the mean maternal buprenorphine dose among infants who required treatment for NAS $(12.1 \mathrm{mg})$ and infants who did not require treatment $(13.9 \mathrm{mg})$, which has been previously reported. ${ }^{3}$ No significant difference was found in the NAS severity of infants born to mothers who were taking buprenorphine before pregnancy (33\% of infants who were treated for NAS) and infants born to mothers who initiated treatment during the course of their pregnancy (35\% of infants who required treatment). This does not support the findings of other authors who reported that, when buprenorphine treatment is initiated before conception, NAS is significantly less frequent than in opioid-dependent women who initiate treatment after conception $(26 \%$ of infants compared with $60 \%$, respectively). ${ }^{20}$

There was no significant relationship between the maternal dose of buprenorphine and the severity of NAS. This paradoxical finding has been reported by others. ${ }^{14,18,21,22}$ Authors of another study found no relationship between the total or cumulative third trimester dose of buprenorphine or dose at delivery and free and total buprenorphine concentrations in the meconium of the infant, suggesting that differences in fetal metabolism or placental transfer may contribute to this finding. ${ }^{22}$ It is also important to note that the prescribed dose and actual dose taken may vary because diversion of buprenorphine is possible.

There was no significant relationship between daily tobacco consumption and NAS severity. Previous studies have found a positive relationship between heavy tobacco use and NAS severity. ${ }^{3,23}$ In this case series, infants born to mothers who used tobacco had a significantly lower birth weight $(P=$ .0136) than infants born to nonusers. This is consistent with the literature pertaining to tobacco use during pregnancy in the non-opioid-dependent population. $^{24,25}$

It is important to note that NAS scoring is highly subjective. The results of this case series may have been impacted by concomitant use of other drugs, especially nicotine, cocaine, other stimulants, and benzodiazepines, because a variety of substances can cause an abstinence syndrome in infants. ${ }^{12}$ Concurrent use of multiple substances by women in this case series was common, and this is consistent with the experience of many previous authors. ${ }^{9,14-16,18,20-22,26}$ 
Buprenorphine is excreted in breast milk at a breast milk to plasma ratio of $1: 1$, but given the poor oral bioavailability of the medication, infant exposure through breast milk should have little impact on NAS scoring. ${ }^{26,27}$ The Center for Substance Abuse Treatment panel recommends breastfeeding for mothers taking buprenorphine unless other contraindications exist. ${ }^{27}$ These recommendations seem to be supported by the results of a small study of 7 breastfeeding women maintained on buprenorphine, which showed that the relative dose per kilogram of body weight in the infant was $<1 \%$ of the dose per body weight of the mother. ${ }^{28}$ Similarly, the range of nor-buprenorphine (a metabolite of buprenorphine) in the mothers' urine ranged from 175 to $933 \mathrm{nmol} / \mathrm{L}$ compared with 1 to $4.8 \mathrm{nmol} / \mathrm{L}$ in the infants. ${ }^{28}$ The infants in this case series were at least if not more likely to be breastfed (70\% at hospital discharge and $50 \%$ at $6-$ to 8 -week follow-up visit) than those in previous studies $(50 \%, 45 \%$, and $21 \%$ as measured during hospitalization). ${ }^{14,18,29}$ In addition, other authors have reported that, of the minority of opioid-dependent women who elected to breastfeed, more than half stopped within 1 week, whereas a much larger percentage of the women in this case series elected to breastfeed until at least their 6- to 8-week follow-up visit. ${ }^{29}$

\section{Conclusions}

The observations regarding the effects of buprenorphine on opioid-dependent pregnant women and their infants made in this case series are similar to those previously reported. Women in this series were more likely to breastfeed their infants than women in previous studies. Further study is needed to determine whether this difference is significant. Maternal polysubstance use (eg, nicotine, benzodiazepines) was common and may have influenced the results (eg, birth weight and NAS scores); infants may have been exposed to more than one substance in utero and may have experienced withdrawal from more than one substance after delivery. Maternal polysubstance use is common in the opioid-dependent pregnant population and also has been reported by other authors.

With grateful appreciation to Greg Wellenius, ScD, Brown University School of Medicine; and Gina Hoang, MD; Greg Feero, MD; and the clinicians and staff of Maine Dartmouth Family Practice and Maine General Medical Center.

\section{References}

1. Bolnick JM, Rayburn WF. Substance use disorders in women: special considerations during pregnancy. Obstet Gynecol Clin North Am 2003;30: 545-58.

2. US Centers for Disease Control and Prevention. Unintentional poisoning deaths-United States, 1999-2004. Available from: http://www.cdc.gov/mmwr/preview/ mmwrhtml/mm5605a1.htm. Accessed October 17, 2010.

3. Fischer G, Johnson RE, Eder H, et al. Treatment of opioid-dependent pregnant women with buprenorphine. Addiction 2000;95:239-44.

4. Kaltenbach K, Berghella V, Finnegan L. Opioid dependence during pregnancy. Obstet Gynecol Clin North Am 1998;25:139-51.

5. Barr GA, Jones K. Opiate withdrawal in the infant. Neurotoxicol Teratol 1994;16:219-25.

6. Mattick RP, Breen C, Kimber J, Davoli M. Methadone maintenance therapy versus no opioid replacement therapy for opioid dependence. Cochrane Database Syst Rev 2009;(3):CD002209.

7. Center for Substance Abuse Treatment. Chapter 13. Medication-assisted treatment for opioid addiction during pregnancy. In: SAMHSA/CSAT treatment improvement protocols. Available from: http://www.ncbi. nlm.nih.gov/bookshelf/br.fcgi?book=hssamhsatip\& part $=$ A83488. Accessed October 17, 2010.

8. Jones HE, Martin PR, Heil SH, et al. Treatment of opioid-dependent pregnant women: clinical and research issues. J Subst Abuse Treat 2008;35:245-59.

9. Jones HE, Johnson RE, Jasinski DR, et al. Buprenorphine versus methadone in the treatment of pregnant opioid-dependent patients: effects on the neonatal abstinence syndrome. Drug Alcohol Depend 2005; 79:1-10.

10. Binder T, Vavrinkova B. Prospective, randomised, comparative study of the effect of buprenorphine, methadone, and heroin on the course of pregnancy, birthweight of newborns, early postpartum adaptation, and course of the neonatal abstinence syndrome (NAS) in women followed up in the outpatient department. Neuro Endocrinol Lett 2008;29:80-6.

11. Johnson RE, Jones HE, Fischer G. Use of buprenorphine in pregnancy: patient management and effects on the neonate. Drug Alcohol Depend 2003;70:S87101.

12. Dunlop AJ, Panjari M, O'Sullivan H, et al. Clinical guidelines for the use of buprenorphine in pregnancy. Fitzroy, Turning Point Alcohol and Drug Centre; 2003.

13. Hamdan AH. Neonatal abstinence syndrome. Available from: http://emedicine.medscape.com/article/ 978763-overview. Accessed October 17, 2010.

14. Fischer G, Ortner R, Rohrmeister K, et al. Methadone versus buprenorphine in pregnant addicts: a 
double-blind, double-dummy comparison study. Addiction 2006;101:275-81.

15. Kayemba-Kay's S, Laclyde JP. Buprenorphine withdrawal syndrome in newborns: a report of 13 cases. Addiction 2003;98:1599-604.

16. Lacroix I, Berrebi A, Chaumerliac M, LapeyreMestre M, Montastruc JL, Damase-Michel C. Buprenorphine in pregnant opioid-dependent women: first results of a prospective study. Addiction 2004; 99:209-14.

17. Kahila H, Saisto T, Kivitie-Kallio S, Haukkamaa M, Halmesmäki E. A prospective study on buprenorphine use in pregnancy: effects on maternal and neonatal outcome. Acta Obstet Gynecol Scand 2007; 86:185-90.

18. Lejeune C, Simmat-Durrand L, Gourarier L, Aubisson S, Groupe d'Etudes Grossesse et Addictions (GEGA). Prospective multicenter observational study of 260 infants born to 259 opiate-dependent mothers on methadone or high-dose buprenorphine substitution. Drug Alcohol Depend 2006;82:250-7.

19. Jones HE, O'Grady K, Dahne J, et al. Management of acute postpartum pain in patients maintained on methadone and buprenorphine during pregnancy. Am J Drug Alcohol Abuse 2009;35:151-6.

20. Kakko J, Heilig M, Sarman I. Buprenorphine and methadone treatment of opiate dependence during pregnancy: comparison of fetal growth and neonatal outcomes in two consecutive case series. Drug Alcohol Depend 2008;96:69-78.

21. Bakstad B, Sarfi M, Welle-Strand GK, Ravndal E. Opioid maintenance treatment during pregnancy: Occurrence and severity of neonatal abstinence syndrome. A national prospective study. Eur Addict Res 2009;15:128-34.
22. Kacinko SL, Jones HE, Johnson RE, Choo RE, Huestis MA. Correlations of maternal buprenorphine dose, buprenorphine, and metabolite concentrations in meconium with neonatal outcomes. Clin Pharmacol Ther 2008;84:604-12.

23. Winklbaur B, Baewert A, Jagsch R, et al. Association between prenatal tobacco exposure and outcome of neonates born to opioid-maintained mothers. Eur Addict Res 2009;15:150-6.

24. Aagaard-Tillery KM, Porter TF, Lane RH, Varner MW, Lacoursiere DY. In utero tobacco exposure is associated with modified effects of maternal factors on fetal growth. Am J Obstet Gynecol 2008;198: 66.e1-6.

25. US Centers for Disease Control and Prevention. Tobacco use and pregnancy. Available from: http://www. cdc.gov/reproductivehealth/tobaccousepregnancy/ index.htm. Accessed October 17, 2010.

26. Johnson RE, Jones HE, Jasinski DR, et al. Buprenorphine treatment of pregnant opioid-dependent women: maternal and neonatal outcomes. Drug Alcohol Depend 2001;63:97-103.

27. US Department of Health and Human Services. Clinical guidelines for the use of buprenorphine in the treatment of opioid addiction. A treatment improvement protocol: TIP 40. Available from: http:// buprenorphine.samhsa.gov/Bup_Guidelines.pdf. Accessed October 17, 2010.

28. Lindemalm S, Nydert P, Svensson JO, Stahle L, Sarman I. Transfer of buprenorphine into breast milk and calculation of infant drug dose. J Hum Lact 2009;25:199-205.

29. Wachman EM, Byun J, Phillip BL. Breastfeeding rates among mothers of infants with neonatal abstinence syndrome. Breastfeed Med 2010;5:159-64. 